\title{
A NOVEL TWO-COMPONENT DECOMPOSITION FOR CO-POLAR CHANNELS OF GF-3 QUAD-POL DATA
}

\author{
E. Kwok ${ }^{1,2, *}$, C. H. Li ${ }^{2}$, Q. H. Zhao ${ }^{1}, \mathrm{Y} \mathrm{Li}^{1}$ \\ ${ }^{1}$ Institute for Remote Sensing Science and Application, School of Geomatics, Liaoning Technical University, Fuxin, China - \\ 755492936@qq.com \\ ${ }^{2}$ Satellite Surveying and Mapping Application Center, National Administration of Surveying, Mapping and Geoinformation of China, \\ Beijing, China
}

Commission III, WG III/2

KEY WORDS: GF-3 satellite, Model-based decomposition, Two-component decomposition, Co-polar data, PolSAR

\begin{abstract}
:
Polarimetric target decomposition theory is the most dynamic and exploratory research area in the field of PolSAR. But most methods of target decomposition are based on fully polarized data (quad pol) and seldom utilize dual-polar data for target decomposition. Given this, we proposed a novel two-component decomposition method for co-polar channels of GF-3 quad-pol data. This method decomposes the data into two scattering contributions: surface, double bounce in dual co-polar channels. To save this underdetermined problem, a criterion for determining the model is proposed. The criterion can be named as second-order averaged scattering angle, which originates from the $\mathrm{H} / \alpha$ decomposition. and we also put forward an alternative parameter of it. To validate the effectiveness of proposed decomposition, Liaodong Bay is selected as research area. The area is located in northeastern China, where it grows various wetland resources and appears sea ice phenomenon in winter. and we use the GF-3 quad-pol data as study data, which which is China's first C-band polarimetric synthetic aperture radar (PolSAR) satellite. The dependencies between the features of proposed algorithm and comparison decompositions (Pauli decomposition, An\&Yang decomposition, Yamaguchi S4R decomposition) were investigated in the study. Though several aspects of the experimental discussion, we can draw the conclusion: the proposed algorithm may be suitable for special scenes with low vegetation coverage or low vegetation in the non-growing season; proposed decomposition features only using co-polar data are highly correlated with the corresponding comparison decomposition features under quad-polarization data. Moreover, it would be become input of the subsequent classification or parameter inversion.
\end{abstract}

\section{INTRODUCTION}

On 10 August 2016 China launched the GF-3 satellite and was put into operation at the end of January, 2017. It is China's first C-band polarimetric synthetic aperture radar (PolSAR) satellite. PolSAR is an advanced radar imaging technology that records more comprehensive backscatter information of a target by acquiring a multi-channel radar signal and is widely used in the field of object recognition, classification, parameters inversion and many other fields. Polarimetric target decomposition remains the most dynamic and exploratory research area in the field of PolSAR data processing. It is to decompose a scattering matrix or a covariance matrix to the linear combinations of some special typical scattering, such as surface scattering, double bounce scattering and volume scattering. Currently, dominated decomposition techniques include $\mathrm{H} / \mathrm{A} / \alpha$ decomposition and Freeman-Durden decomposition(Lee et al, 2009). But most methods of target decomposition are based on fully polarized data (quad pol) and seldom utilize dual-polar data for target decomposition. Studies that highlight the benefit and applicability of $\mathrm{HH} / \mathrm{VV}$-polarized data are very small(Ullmann et al, 2016). It may be a pity for a number of dual-channels SAR data to make use of target decomposition theory. Obviously, three dual polarization modes $(\mathrm{HH} / \mathrm{HV}$, $\mathrm{VV} / \mathrm{VH}$ and $\mathrm{HH} / \mathrm{VV}$ ) are presented in the combination of dualpolar data, but only co-polarization mode (HH/VV) can generate a second order coherency matrix, which has physical scattering meanings and no assumption of reciprocity. So copolarized channels data is served as the research data source, and then analyzes the decomposition of its scattering mechanism.

\section{STUDY AREA AND DATA DESCRIPTION}

We select Liaodong Bay in winter as the research area. The area is located in northeastern China, bordered by the Bohai Sea to the south, with wetland resources such as herbagedominated marsh, intertidal marshes and shallow beach and sea ice in the southern coastal area in winter. And we use a GaoFen-3 quad-pol data as research data. GaoFen-3 is China's first C-band polarimetric synthetic aperture radar (PolSAR) satellite. Data details are given in Table 1. the incidence angle range and the mean NESZ values are presented. The study area is located in the Liaohe River delta of Panjin City in northeast of China, centered at $41.00^{\circ} \mathrm{N}$ and $121.50^{\circ} \mathrm{E}$, as shown in Figure 1(a) .

A fully polarimetric SAR image in QPSI mode with a central incidence angle of $38^{\circ}$ were acquired at January 23, 2017. The sample spacing of the single look complex (SLC) SAR data is $4.7 \mathrm{~m}$ (range) by $4.9 \mathrm{~m}$ (azimuth). The PauliRGB images composited by $\mathrm{S}_{\mathrm{HH}}-\mathrm{S}_{\mathrm{VV}}$ in red, $\mathrm{S}_{\mathrm{HH}}+\mathrm{S}_{\mathrm{VV}}$ in blue, and $\mathrm{S}_{\mathrm{HV}}$ in green is shown in Figure 1(b). 


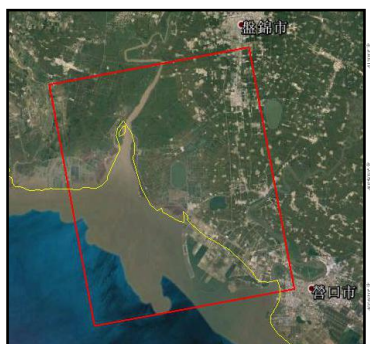

(a) location of study area

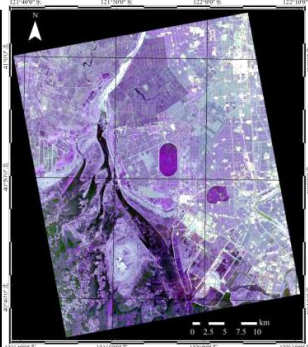

(b) Pauli decomposition map of study area

Figure1. study area

\section{METHODOLOGY}

\subsection{Pre-processing for PolSAR data}

Firstly, GF-3 PolSAR data (from its single-look complex product) is pre-processed, including radiometrically calibration, multi-looking processing $(5 \times 5)$, refined Lee filtering $(7 \times 7)$ and geocoding; Secondly, second-order coherency matrix $T_{2}$ are generated based on sinclair scattering matrix; Thirdly, two component decomposition is operated based on the coherency matrix; Finally, analysis and discuss the decomposition of the results to demonstrate its validity.

\subsection{Coherency matrix $\mathrm{T} 2$ of $\mathrm{HH} / \mathrm{VV}$ data}

Similarity with full-polarized SAR data, $\mathrm{HH} / \mathrm{VV}$ dualpolar information can be expressed by the coherency matrix $T_{2}$ (equation(1,2)) which can evaluate the second-order statistics of the scattering matrix $S$. coherency matrix $T_{2}$ is derived by multiplying the two-component Pauli target vector $k_{\tau}$,

$$
T_{2}=k_{\tau} k_{\tau}^{* \mathrm{~T}}=\left[\begin{array}{ll}
T_{11} & T_{12} \\
T_{21} & T_{22}
\end{array}\right]
$$

where

$$
k_{\tau}=\frac{1}{\sqrt{2}}\left(\begin{array}{c}
S_{h h}+S_{v v} \\
S_{h h}-S_{v v}
\end{array}\right)
$$

${ }^{* \mathrm{~T}}$ represent conjugate transpose operation. In practise, $T_{2}$ can be obtained by

$$
\left\langle T_{2}\right\rangle=\frac{1}{2}\left\langle\left[\begin{array}{cc}
\left|S_{h h}+S_{v h}\right|^{2} & \left(S_{h h}+S_{v v}\right)\left(S_{h h}-S_{v v}\right)^{*} \\
\left(S_{h h}-S_{v v}\right)\left(S_{h h}+S_{v v}\right)^{*} & \left|S_{h h}-S_{v h}\right|^{2}
\end{array}\right]\right)
$$
process.

\subsection{Model-based decomposition of $\mathbf{T}_{2}$}

A straight forward adaption of this approach for HH/VVpolarized SAR data is the decomposition of the span into the scattering power components of surface $\left(P_{\mathrm{s}}\right)$, double bounce $\left(P_{\mathrm{d}}\right)($ see equation $(3))$ : as we know, the estimation of the two component's parameters is based on the co-polarized information alone, so they can be fully extracted; for volume scattering, in theory, we can extract its co-polarized component, here, we ignore it because it is very small.

$$
T_{2}=T_{s}^{H V}+T_{d}^{H V}=f_{s}\left[\begin{array}{cc}
1 & \beta^{*} \\
\beta & |\beta|^{2}
\end{array}\right]+f_{d}\left[\begin{array}{cc}
\mid \alpha^{2} & \alpha \\
\alpha^{*} & 1
\end{array}\right]
$$

From the above equation, the first right term is the surface scattering, the second right term is the double-bounce scattering. Since the number of unknown variables in the decomposition model is one more than the number of equations, that is, the problem is underdetermined. To save this problem, a criterion for determining the model is proposed. It is based on the average scattering angle $\alpha$ which is a parameter in classical $H / A / \alpha$ decomposition. It can be used to recognize the main scattering mechanism, i.e., surface scattering (about $0^{\circ}$ ), volume scattering (about $45^{\circ}$ ) and double bounce scattering (about $90^{\circ}$ ). Accordingly, the criterion is that when $\alpha>45^{\circ}$, it is considered as double bounce scattering dominated, surface scattering as secondary scattering; $\alpha<45^{\circ}$, surface scattering is dominant and secondary scattering is double bounce scattering(Cloude et al, 1996).

$T_{2}$ can be decomposed into

$$
T_{2}=\sum_{i=1}^{2} \lambda_{i} u_{i} u_{i}^{\mathrm{H}}
$$

where $\lambda_{i}(i=1,2)$ are the eigenvalues of $\left\langle T_{2}>\right.$, with $\lambda_{1} \geq \lambda_{2}$. $\boldsymbol{u}_{i}=e^{j \varphi i}\left[\begin{array}{cc}\cos \alpha & \sin \alpha \cos \beta e^{i \delta i}\end{array}\right]^{\mathrm{T}}$ are eigenvectors, $\alpha_{i}$ denote the scattering mechanisms of the target, $\beta_{i}$ are the orientation angles, and $\varphi_{i}$ and $\delta_{i}$ are the phases. where $\alpha$ is a parameter of $H / \alpha$ decomposition(Ji et al, 2015). It represents average scattering angle, it can be written as:

$$
\alpha=\sum_{i=1}^{2} P_{i} \alpha_{i}=\frac{\lambda_{1}}{\lambda_{1}+\lambda_{2}} \alpha_{1}+\frac{\lambda_{2}}{\lambda_{1}+\lambda_{2}} \alpha_{2}
$$

For $H / \alpha$ decomposition of the second-order coherent matrix, the efficiency of the algorithm is significantly reduced. In this paper, an alternative parameter $A P$ of the second-order averaged scattering angle $\alpha$ is introduced, which is essentially a measure of the relationship between the main elements of two second-order coherency matrices.

$$
A P=\frac{T_{22}}{T_{11}+T_{22}}=\frac{T_{22}}{\text { span }}
$$

Case 1: when $\alpha<45^{\circ}$ or $\mathrm{AP}<0.5, \alpha=0$; we regard that surface scattering is dominant, the solution can be written by

$$
\begin{gathered}
f_{s}=T_{11} \\
f_{d}=T_{22}-\left(\frac{\left|T_{12}\right|^{2}}{T_{11}}\right) \\
\beta^{*}=\frac{T_{12}}{T_{22}}
\end{gathered}
$$

So the scattering power components $P_{\mathrm{s}}$ and $P_{\mathrm{d}}$ can be derived

$$
P_{s}=f_{s}\left(1+|\beta|^{2}\right), \quad P_{d}=f_{d}
$$

Case 2: when $\alpha>45^{\circ} / \mathrm{CP}>0.5, \beta^{*}=0$; double-bounce scattering is dominant.

$$
f_{d}=T_{22}
$$




$$
\begin{gathered}
f_{s}=T_{11}-\left(\frac{\left|T_{12}\right|^{2}}{T_{22}}\right) \\
\alpha=\frac{T_{12}}{T_{22}}
\end{gathered}
$$

So the scattering power components $P_{\mathrm{s}}$ and $P_{\mathrm{d}}$ can be derived:

$$
P_{s}=f_{s}, \quad P_{d}=f_{d}\left(1+|\alpha|^{2}\right)
$$

\section{RESULTS \& DISCUSSION}

\subsection{Proposed decomposition results}

The first two figures are the intensity plots of two co-polarized channels. It can be seen that the polarizations of the copolarized polarizations are basically the same. The image above is the decomposition result of the algorithm in the figure. Is the secondary scatter power map, Figure 2 is a two-scatter RGB composite map where the red channel is second-order scattering, the blue channel is the surface scattering and the green channel is set to zero; in general, the surface scattering component is in the natural medium The existence is more common, especially in the winter phase of the selected phase in this paper, the solidification of the land plate becomes smaller and the roughness becomes smaller. Therefore, the surface scattering component accounts for a larger proportion of freshwater swamps (reeds). Secondary scattering mainly occurs in those vertical structural areas, such as urban areas typified by manmade structures, or secondary scattering occurs when tall plants are in the growing season. It can be seen that in this scenario, the high secondary scattering components are mainly concentrated in the urban area (i.e the red area in Figure 4).

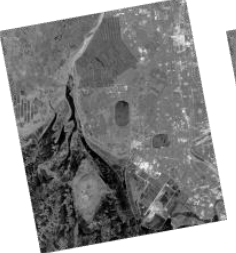

(a). $\mathrm{Chh}$

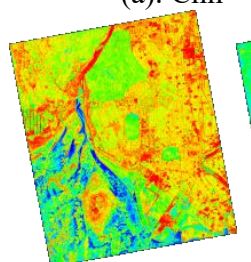

(c). Ps

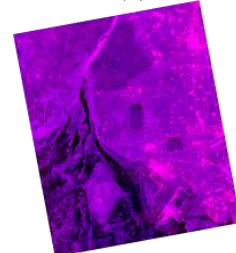

(d). $P d$

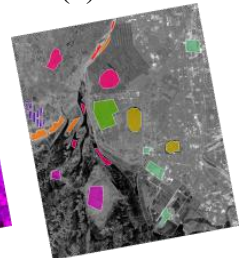

(e). RGB display of proposed decomposition

(f) 10 types of samples in the HH's intensity Figure 2. polarimetric features

\subsection{Three criterions Analysis of selecting model}

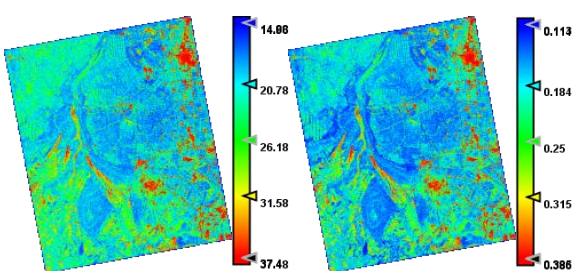

Figure 3. mean scattering angle $\alpha$ Figure 4 . alternative parameter(AP)

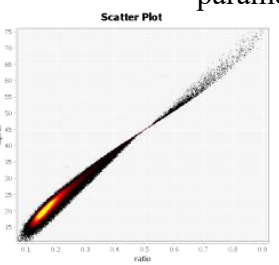

Figure 5. 2-axis plot of $\alpha$ and AP

The above figures show the second-order mean scattering angle and the alternative parameter map. It can be seen from the above figure that the substitution parameters and the scattering angle are basically the same in describing the ground-based scattering mechanism. Figure 3,4 shows the results of the twoparameter mapping. It can be seen that the scattering When $\alpha=$ $45, \mathrm{AP}=0.5$, all the data points are divided into two spaces by the point. Most of the points are distributed in the lower left space (ie, $\alpha<45^{\circ}$, AP $<0.5$ ). The types are mainly surface scattering. The data point scattering types distributed in the upper right belong to the second scattering. Generally speaking, the two parameters basically maintain a linear relationship. Since the calculation of the scattering angle requires the eigen decomposition of the coherence matrix, which leads to the reduction of the computational efficiency, the alternative parameters are more suitable than the scattering angle in the actual calculation as a criterion and can be used as an effective indicator of the initial judgment of the scattering mechanism.

\subsection{Correlation}

\begin{tabular}{ll}
\hline features & decomposition \\
\hline $\begin{array}{l}\text { surface, } \\
\text { double-bounce } \\
\text { surface, } \\
\text { double-bounce } \\
\text { surface, } \\
\text { double-bounce }\end{array}$ & Pauli \\
\hline Table 1. comprision decomposition features
\end{tabular}

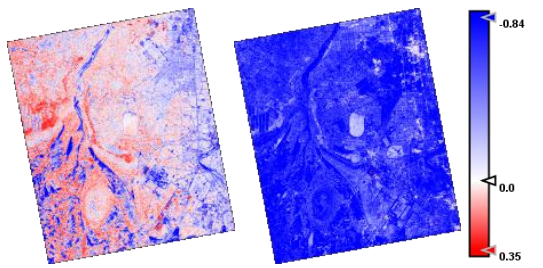

Figure 6. difference between proposed's Ps and Yamaguchi's Ps

Figure 7. difference between proposed's $\mathrm{Pd}$ and Yamaguchi's Pd

In order to verify the validity of the proposed algorithm, we choose An \& Yang decomposition and Yamaguchi S4R fourcomponent decomposition as the comprision algorithm in the fully-polarized SAR data.(see Table 1) Since only two scattering types are decomposed in this paper, so we only the 
corresponding components of the comparison algorithm are analyzed here. The above figure shows the difference between the surface scattering component and the second-order scattering component of the decomposition of Yamaguchi S4R, respectively, and the decomposition component of this paper. An \& Yang decomposition is similar to the difference between this method and the general decomposition. For surface scattering, the urban area and the sea-ice boundary area are underestimated, but the wetland area (salt marshes, freshwater swamps, winter paddy field, etc.) and sea ice are often overestimated; for the second scattering, this algorithm generally underestimates its contribution, but still within the error range.

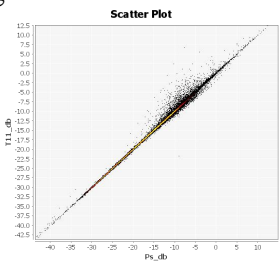

(a) Ps:Pauli-proposed

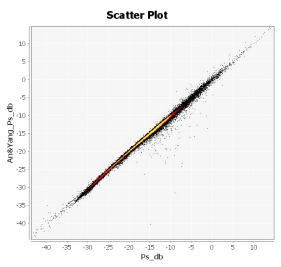

(b) Ps:An-proposed

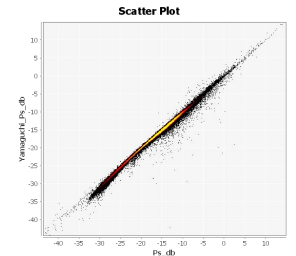

(c) Ps:Yamaguchi-proposed

Figure 8. different surface scattering comprision

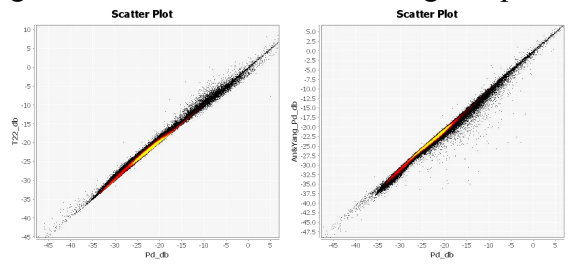

$\begin{array}{lll}\text { (a) Pd:Pauli-proposed } & \text { (b) Pd:An-proposed }\end{array}$

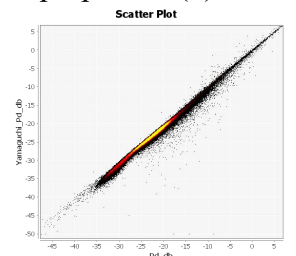

(c) Pd:Yamaguchi-proposed

Figure 9. different double bounce scattering comprision

Since both scattering mechanisms are theoretically extracted from co-polarized channels (i. e, $\mathrm{T}_{11}, \mathrm{~T}_{22}$ ), and they should not be related to the order of decomposition. On the other hand, the two decompositions consider the influence of the orientation angle rotation, so the main diagonal of the coherence matrix element (power) change, and it makes that the two types of scattering under full polarization conditions were completely extracted. Figure 8,9 are two-dimensional scatter plots of the corresponding components of the algorithm and other three contrast decompositions. It can be seen that the distribution of off-diagonal scatter points can reflect the degree of difference of the selected parameters. the farther away from the diagonal means that the difference is the greater. For the surface scattering components, the difference between proposed's Ps and the Ps of Pauli is smaller than the difference between proposed's Pd and the Pd of An and Yamaguchi, which basically distributes in the diagonal. From Fig.5(a), the nondiagonal point distribution is medium power intensity range, there are more points below the diagonal point, indicating that as such points have the feature that $\alpha>45^{\circ}$, model was selected as the secondary scattering dominated. Figure $8(\mathrm{~b})$ and Figure 8(c) show the difference between proposed's Ps and An, Yamaguchi decomposition. Obviously, there are a linear correlation as a whole, of which An decomposition is smaller than that of Yamaguchi decomposition. The reason that cause the difference of similar scattering power is the difference of the volume scattering models extracted by the two (the latter is more complicated and fine). For the double bounce scattering components, the proposed $\mathrm{Pd}$ is more different than the comparison algorithm, but basically accord with the linear relationship.

\subsection{Backscattering Analysis}

This paper investigates the polarization scattering of several typical ground objects in coastal wetlands in winter. Fig.3(f) is the selected samples of each object on the HH channel's intensity image. The features of the study area can be divided into 10 categories, namely urban areas(UA), freshwater swamps(FS), salt marshes(SM), winter paddy fields(WP), reservoirs(RE), seawater(SW), sea ice(SI), river ice(RI), sea farms(SF), light beach(LB).

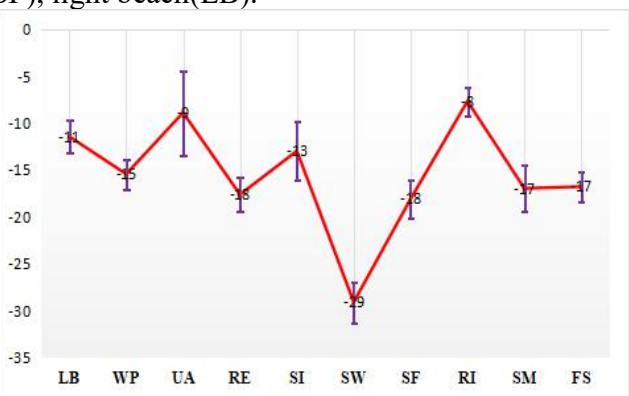

Figure 10. surface scattering of each sample

From Figure 10, the scattering coefficient of seawater is the lowest $(-29 \pm 2.2 \mathrm{~dB})$, the highest scattering coefficient is river ice $(-8 \pm 1.5 \mathrm{~dB})$ or urban area $(-9 \pm 4.5 \mathrm{~dB})$. Similarly, as part of the water system, the range of sea ice scattering is $-13 \pm 3.1$ $\mathrm{dB}$, so we consider that river ice may has more roughness than sea ice. and the range of reservoir scattering is consistent with that of sea farms; the scattering of the two typical wetland types (freshwater marsh and saltwater marsh) is comparable with only minor differences. However, given the winter time phase selected, the vegetation is in the non-growing seasons, which has similar moisture condition. therefore, freshwater marsh can not be distinguish with saltwater marsh.

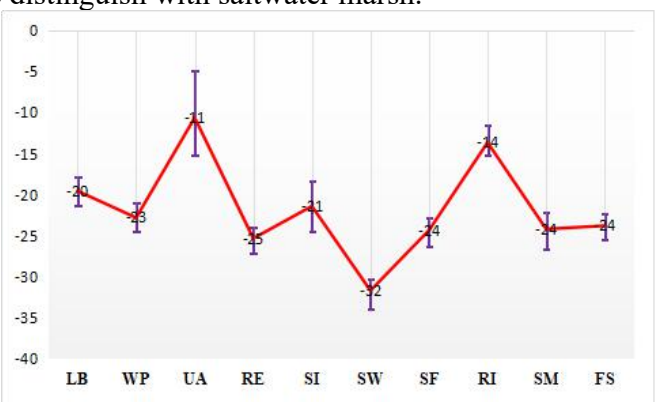

Figure 11. double bounce scattering of each sample

The polarimetric response of this algorithm for each type of object as shown in Figure 10,11, we find that the two scattering components of the polarization response are similar for different land covers. differences in surface scattering of different land covers are more larger. similar types of wetland can not be distinguished only using polarimetric scattering features. The 
scattering coefficients of light beach is very similar with winter paddy fields, in addition, it it not distinguished between freshwater swamps and salt marshes. urban area has the strongest backscattering coefficient(include Ps and Pd), which range is more concentrated. it may cuased by the relatively smooth surface of such artificial area. we can see from Figure 11 , the urban area has more dihedral reflector structure, so the secondary scattering component is strong. It often used as an indicator of detection of urban areas.

\section{CONCLUSION}

We put forward to a novel model-based decomposition of $\mathrm{HH} / \mathrm{VV}$ dual-pol data which can inculde two typical scattering model. it can be used to monitoring of urban or residential area And it also shows that HH/VV information, which main include surface and double-bounce scattering information, plays a key role in quad-pol mode. In addition, we analysis sea ice ( its thickness $<30 \mathrm{~cm}$ ), varies of wetlands, and urban areas's backscattering characteristic of co-polar channels in winter.

\section{ACKNOWLEDGEMENTS}

This study was supported by the National Natural Science Foundation of China (No. 41271435, 41301479) and the Natural Science Foundation of Liaoning, China (No.2015020090). The authors would like to thank the Satellite Surveying and Mapping Application Centor (NASG) for their support in providing GaoFen-3 quad-pol data.

\section{REFERENCES}

An W.; Cui Y.; Yang J, 2010. Three-Component Model-Based Decomposition for Polarimetric SAR Data[J]. IEEE Trans. Geosci.Remote Sens, 48(6), pp. 2732-2739.

A. Sato, Y. Yamaguchi, G. Singh, and S.-E. Park, 2012. Fourcomponent scattering power decomposition with extended volume scattering model, IEEE Geosci., Remete Sens. Letters, 9(2), pp. 166-170.

Cloude, S.R.; Pottier, E, 1996. A review of target decomposition theorems in radar Polarimetry. IEEE Trans. Geosci.Remote Sens, 34(2), pp. 498-518.

Cloude, S.R., 2009. Polarisation: Applications in Remote Sensing. Oxford University Press, Oxford, pp. 1-453.

Ji, K.; Wu, Y, 2015. Scattering Mechanism Extraction by a Modified Cloude-Pottier Decomposition for Dual Polarization SAR. Remote Sens, 7(6), pp. 7447-7470.

Lee, J.-S.; Pottier, E, 2009. Polarimetric Radar Imaging: From Basics to Applications. CRC Press, Boca Raton, pp. 1-422.

Ullmann, T.; Schmitt, A.; Jagdhuber, 2016. Two Component Decomposition of Dual Polarimetric HH/VV SAR Data: Case Study for the Tundra Environment of the Mackenzie Delta Region, Canada. Remote Sens. 8(12), pp. 1027-1050. 